\title{
Analysis of the barycentric interpolation collocation scheme for the Burgers equation
}

\author{
Yudie Hu, Ao Peng, Liquan Chen, Yanlei Tong, Zhifeng Weng* \\ School of Mathematical Sciences, Huaqiao University, Quanzhou, Fujian 362021 China \\ *Corresponding author, e-mail: zfwmath@163.com
}

Received 3 Feb 2021

Accepted 29 May 2021

\begin{abstract}
In this paper, we propose the barycentric interpolation collocation method for the Burgers equation. Firstly, Burgers equation is transformed into the heat conduction equation by the Hopf-Cole transformation. Then we use the barycentric interpolation collocation method to discretize the equation in the time and space directions. The collocation scheme of the Burgers equation is constructed and the corresponding linear algebraic equations are derived. Moreover, the consistency analysis of barycentric Lagrange interpolation method is given. Numerical examples validate the efficiency of our scheme.
\end{abstract}

KEYWORDS: Burgers equation, Hopf-Cole transformation, heat conduction equation, consistency analysis, barycentric interpolation collocation method

MSC2010: 65M70 65L20

\section{INTRODUCTION}

In 1915, Bateman [1] proposed the Burgers equation in nonlinear partial differential equations when studying fluid motion. Burgers equation has the characteristics of both the wave equation and heat conduction equation. It maintains the basic characteristics of the Navier-Stokes equation in fluid dynamics and is an ideal simplified model for complex fluid dynamics problems. Burgers equation plays an important role in many fields such as fluid mechanics, quantum field theory, transport and dispersion of pollutants in rivers and sediment transport, etc. Therefore, it is of great significance to discuss its numerical solution.

Due to the shock wave phenomenon of Burgers equation, it is difficult to obtain the analytical solutions. Therefore, it is necessary to discuss its numerical solution. At present, the main methods are the finite difference method, finite element method and so on. The Hopf-Cole transformation was firstly proposed by Hopf [2]. Kadalbajoo et al [3] proposed the Hopf-Cole transformation to the heat conduction equation with the Neumann boundary, and gave an unconditionally stable second-order implicit difference scheme. Ram [4] developed Haar wavelet basis functions and Euler's implicit scheme to solve Burgers equation. Zhang et al [5] presented a nonlinear implicit difference scheme to solve Burgers equation and gave the corresponding error analysis. Sari [6] developed a cubic B-spline Galerkin finite element method combined with operator splitting technique to solve Burgers equation. Wang et al [7] introduced the weak Galerkin (WG) finite element method to study a class of time fractional generalized Burgers equation.

The above methods are all based on mesh generation for the Burgers equation. Recently, some studies [8-10] proposed the barycentric interpolation collocation method. Many researchers extended this method to solve boundary value problems [11] and various differential/integral equations, such as the coupled reaction-diffusion equation [12], autoconvolution Volterra integral equations [13], linear and nonlinear high-dimensional Fredholm integral equations [14], generalized Abel integral equations [15] and parabolic partial differential equations $[16,17]$. The barycentric interpolation collocation method is a high-precision numerical scheme that does not depend on mesh generation. When the number of nodes increases, the approximate function constructed by the Lagrange interpolation formula will lead to Runge phenomenon, which has great numerical instability. Fortunately, if the nodes obey the density proportion $\left(1-x^{2}\right)^{-1 / 2}$ such as the families of Chebyshev points that are the simplest clustered point sets, the barycentric Lagrange interpolation has a good numerical stability. 
To our knowledge, the works of the error analysis for barycentric interpolation collocation method are relatively sparse. Recently, Yi and Yao [18] put forward a steady barycentric Lagrange interplotaion method and presented error analysis of system for solving the time-fractional telegraph equation. Li [19] provided the error estimate of the barycentric rational interpolation collocation method for the heat conduction equation. Based on the above work, we focus on the collocation scheme of the Burgers equation and transform it into a linear problem through the Hopf-Cole transformation, which avoids the solution of nonlinear discretized equations. Moreover, we give a consistency analysis of the collocation scheme.

\section{BARYCENTRIC INTERPOLATION COLLOCATION METHOD}

\section{Hopf-Cole transformation of Burgers equation}

In this article, we consider the Burgers equation with Dirichlet boundary and initial conditions as follows

$$
\left\{\begin{array}{rlrl}
\frac{\partial u}{\partial t}+u \frac{\partial u}{\partial x}=v \frac{\partial^{2} u}{\partial x^{2}}, & & a<x<b, 0<t \leqslant T, \\
u(x, 0)=g(x), & \quad a \leqslant x \leqslant b, \\
u(0, t)=\theta(t), & 0 \leqslant t \leqslant T, \\
u(1, t)=\omega(t), & 0 \leqslant t \leqslant T .
\end{array}\right.
$$

where $v=1 / \operatorname{Re}>0$ is a viscosity coefficient, and $g(x), \theta(t), \omega(t)$ are given functions. The Hopf-Cole transformation [2] is as follows

$$
u(x, t)=-2 v \frac{\varphi_{x}}{\varphi} .
$$

The nonlinear Burgers equation is transformed into a linear heat conduction equation

$$
\left\{\begin{array}{c}
\frac{\partial \varphi}{\partial t}=v \frac{\partial^{2} \varphi}{\partial x^{2}}, \quad 0<x<1,0<t \leqslant T, \\
\varphi(x, 0)=\mathrm{e}^{-\frac{1}{2 v} \int_{0}^{x} g(s) \mathrm{d} s}, \quad 0 \leqslant x \leqslant 1, \\
\theta(t) \varphi(0, t)+2 v \varphi_{x}(0, t)=0, \quad 0 \leqslant t \leqslant T, \\
\omega(t) \varphi(1, t)+2 v \varphi_{x}(1, t)=0, \quad 0 \leqslant t \leqslant T .
\end{array}\right.
$$

To find the numerical solution of the Eq. (3), the numerical solution of the original Burgers equation can be obtained by the Hopf-Cole inverse transformation.

\section{Barycentric Lagrange interpolation with the Chebyshev points}

Let $p(x)$ approximate the function $\varphi(x)$ on interval $[a, b]$ with partition $a=x_{0}<x_{1}<\cdots<x_{m}=b$. The
Lagrange interpolation function of $\varphi(x)$ is given as

$$
p(x)=\sum_{j=0}^{m} L_{j}(x) \varphi_{j},
$$

where

$$
L_{j}(x)=\prod_{i=0, i \neq j}^{m} \frac{\left(x-x_{i}\right)}{\left(x_{j}-x_{i}\right)}, \quad j=0,1, \ldots, m,
$$

$\sum_{j=0}^{m} L_{j}(x)=1$, and $\varphi_{j}=\varphi\left(x_{j}\right)$.

Let $l(x)=\left(x-x_{0}\right)\left(x-x_{1}\right) \cdots\left(x-x_{m}\right)$ and $w_{j}=1 / \prod_{i=0, i \neq j}^{m}\left(x_{j}-x_{i}\right), j=0,1, \ldots, m$, then the interpolation basis function can be expressed as

$$
L_{j}(x)=l(x) \frac{w_{j}}{x-x_{j}}, \quad j=0,1, \ldots, m .
$$

Taking Eq. (5) into (4), we have

$$
p(x)=l(x) \sum_{j=0}^{m} \frac{w_{j}}{x-x_{j}} \varphi_{j} .
$$

Combining the Eqs. (4)-(6), the barycentric Lagrange interpolation function is obtained as follows

$$
p(x)=\frac{\sum_{j=0}^{m} \frac{w_{j}}{x-x_{j}} \varphi_{j}}{\sum_{j=0}^{m} \frac{w_{j}}{x-x_{j}}} .
$$

The barycentric Lagrange interpolation is illconditioned for equidistant nodes. Choosing a node family with a density ratio of $\left(1-x^{2}\right)^{-1 / 2}$ can make it numerically stable. The simplest node distribution is the Chebyshev point family.

Therefore we choose the Chebyshev points

$$
x_{j}=\cos \left(\frac{j}{m} \pi\right), \quad j=0,1, \ldots, m
$$

to ensure the numerical stability of the Barycentric Lagrange interpolation. Then we find

$$
w_{j}=(-1)^{j} \vartheta_{j}, \quad \vartheta_{j}= \begin{cases}1 / 2, & j=0 \text { or } n \\ 1, & \text { otherwises. }\end{cases}
$$

\section{BARYCENTRIC INTERPOLATION COLLOCATION SCHEME OF HEAT CONDUCTION EQUATION}

For distinct nodes $\left(x_{0}, t\right),\left(x_{1}, t\right), \ldots,\left(x_{m}, t\right)$, the value of the unknown function $\varphi(x, t)$ of the node $(x, t)$ is expressed as $\varphi\left(x_{i}, t\right), i=0,1, \ldots, m$.

The Barycentric Lagrange interpolation function is

$$
\varphi(x, t)=\sum_{j=0}^{m} L_{j}(x) \varphi_{j}(t),
$$


taking the points $x_{0}, x_{1}, \ldots, x_{m}$ into (10) and taking (10) into (3), we have for $i=0,1, \ldots, m$,

$$
\sum_{j=0}^{m} L_{j}\left(x_{i}\right) \dot{\varphi}_{j}(t)=v \sum_{j=0}^{m} L_{j}^{\prime \prime}\left(x_{i}\right) \varphi_{j}(t)
$$

where $\dot{\varphi}_{j}(t)$ is the first order derivative of $\varphi_{j}(t)$. Then we have

$$
\begin{array}{r}
\sum_{j=0}^{m} \delta_{i j} \dot{\varphi}_{j}(t)=v \sum_{j=0}^{m} C_{i j}^{(2)}\left(x_{i}\right) \varphi_{j}(t), \\
\delta_{i j}=L_{j}\left(x_{i}\right)= \begin{cases}1, & i=j, \\
0, & i \neq j,\end{cases}
\end{array}
$$

where $C^{(p)}=\left(C_{i j}^{(p)}\right)_{m \times m}$ is the $p$-order differential matrix of the function. By deriving $L_{j}(x)$, and then using mathematical induction, the recursive formula of $C^{(p)}$ can be obtained.

$$
\left\{\begin{array}{l}
C_{i j}^{(p)}=p\left(C_{i i}^{(p-1)} C_{i j}^{(1)}-\frac{C_{i j}^{(p-1)}}{x_{i}-x_{j}}\right), \quad i \neq j, \\
C_{i i}^{(p)}=-\sum_{j=0, j \neq i}^{m} C_{i j}^{(p)} .
\end{array}\right.
$$

Combining the Eqs. (12) and (13), the matrix forms can be expressed as follows

$$
\left(\begin{array}{c}
\dot{\varphi}_{0}(t) \\
\vdots \\
\dot{\varphi}_{m}(t)
\end{array}\right)=v\left(\begin{array}{ccc}
C_{00}^{(2)} & \cdots & C_{0 m}^{(2)} \\
\vdots & \ddots & \vdots \\
C_{m 0}^{(2)} & \cdots & C_{m m}^{(2)}
\end{array}\right)\left(\begin{array}{c}
\varphi_{0}(t) \\
\vdots \\
\varphi_{m}(t)
\end{array}\right) .
$$

Similarly as the discrete formula as space variable $x$, we get the discrete formula of time variable $t$ as

$$
\begin{gathered}
\varphi_{i}\left(t_{j}\right)=\varphi\left(x_{i}, t_{j}\right)=\varphi_{i j}, \\
i=0,1, \ldots, m, j=0,1, \ldots, n, \text { and } \\
\varphi_{i}(t)=\sum_{k=0}^{n} L_{k}(t) \varphi_{i k}, \quad i=0,1, \ldots, m .
\end{gathered}
$$

Taking Eq. (15) into Eq. (14), and taking the points $t_{0}, t_{1}, \ldots, t_{n}$ into Eq. (14), we have for $j=0,1, \ldots, n$

$$
\begin{aligned}
& \left(\begin{array}{c}
\sum_{k=0}^{n} \dot{L}_{k}\left(t_{j}\right) \varphi_{0 k} \\
\vdots \\
\sum_{k=0}^{n} \dot{L}_{k}\left(t_{j}\right) \varphi_{m k}
\end{array}\right)= \\
& v\left(\begin{array}{ccc}
C_{00}^{(2)} & \cdots & C_{0 m}^{(2)} \\
\vdots & \ddots & \vdots \\
C_{m 0}^{(2)} & \cdots & C_{m m}^{(2)}
\end{array}\right)\left(\begin{array}{c}
\sum_{k=0}^{n} L_{k}\left(t_{j}\right) \varphi_{0 k} \\
\vdots \\
\sum_{k=0}^{n} L_{k}\left(t_{j}\right) \varphi_{m k}
\end{array}\right)
\end{aligned}
$$

Eq. (16) can be written as matrix form

$$
\left(I_{m+1} \otimes D^{(1)}\right) \varphi=v\left(C^{(2)} \otimes I_{n+1}\right) \varphi,
$$

which can be written as the simple forms as

$$
L \varphi=0,
$$

where $L=\left(I_{m+1} \otimes D^{(1)}\right)-v\left(C^{(2)} \otimes I_{n+1}\right)$, and $\otimes$ is the Kronecher product of matrix, $\dot{L}_{k}\left(t_{j}\right)=D_{j k}^{(1)}, \varphi=$ $\left[\varphi_{00}, \ldots, \varphi_{0 n}, \varphi_{10}, \ldots, \varphi_{1 n}, \ldots, \varphi_{m 0}, \ldots, \varphi_{m n}\right]^{\mathrm{T}}$.

The discrete form of the initial condition is

$$
\varphi\left(x_{i}, 0\right)=\varphi_{i 0}=\psi\left(x_{i}\right), \quad i=0,1, \ldots, m .
$$

The corresponding matrix form is

$$
\begin{array}{r}
\left(\begin{array}{c}
\varphi\left(x_{0}, 0\right) \\
\varphi\left(x_{1}, 0\right) \\
\vdots \\
\varphi\left(x_{m}, 0\right)
\end{array}\right)=\left(\begin{array}{c}
\varphi_{00} \\
\varphi_{10} \\
\vdots \\
\varphi_{m 0}
\end{array}\right)=\left(I_{m+1} \otimes e_{n+1}^{1}\right) \varphi \\
=\psi\left(x_{i}\right)=\left(\begin{array}{c}
\psi\left(x_{0}\right) \\
\psi\left(x_{1}\right) \\
\vdots \\
\psi\left(x_{m}\right)
\end{array}\right),
\end{array}
$$

where $e_{n+1}^{1}$ represents the first row of the identity matrix of order $n+1, I_{m+1}$ represents the identity matrix of order $m+1$.

The discrete form of the boundary conditions is, for $0 \leqslant k \leqslant n$,

$$
\left\{\begin{array}{c}
\theta\left(t_{k}\right) \varphi\left(x_{0}, t_{k}\right)+2 v \sum_{j=0}^{m} L_{j}^{(1)}\left(x_{0}\right) \varphi\left(x_{0}, t_{k}\right)=0, \\
\omega\left(t_{k}\right) \varphi\left(x_{m}, t_{k}\right)+2 v \sum_{j=0}^{m} L_{j}^{(1)}\left(x_{m}\right) \varphi\left(x_{m}, t_{k}\right)=0 .
\end{array}\right.
$$

Then Eq. (20) can be written as matrix form

$$
\begin{aligned}
& C_{1} \otimes \theta \cdot \varphi+2 v\left(C_{1} \otimes I_{n+1}\right) \cdot\left(C^{(1)} \otimes I_{n+1}\right) \cdot \varphi=0, \\
& C_{2} \otimes \omega \cdot \varphi+2 v\left(C_{2} \otimes I_{n+1}\right) \cdot\left(C^{(1)} \otimes I_{n+1}\right) \cdot \varphi=0,
\end{aligned}
$$
where

$$
\begin{aligned}
& C_{1}=\left(\begin{array}{llll}
1 & & & \\
& 0 & & \\
& & \ddots & \\
& & & 0
\end{array}\right), C_{2}=\left(\begin{array}{llll}
0 & & & \\
& \ddots & & \\
& & 0 & \\
& & & 1
\end{array}\right) \\
& \theta=\left(\begin{array}{ccc}
\theta\left(t_{0}\right) & & \\
& \ddots & \\
& & \theta\left(t_{n}\right)
\end{array}\right), \omega=\left(\begin{array}{ccc}
\omega\left(t_{0}\right) & & \\
& \ddots & \\
& & \omega\left(t_{n}\right)
\end{array}\right) \text {. }
\end{aligned}
$$

To deal with the boundary conditions of the Eq. (3), we derived the matrix form (21)-(22) for 
the discretization of boundary conditions. Here we use the replacement method to deal with the boundary conditions. We respectively replace the first $n+1$ rows of Eq. (21) and the last $n+1$ rows of Eq. (22) with the corresponding number of rows in Eq. (18) to deduce the linear algebraic equation system.

\section{CONSISTENCY ANALYSIS}

In this part, we present consistency estimates of the scheme (18) with the collocation method. According to interpolation remainder theorem, we have

$$
e(x):=\varphi(x)-p(x)=\frac{\varphi^{(m+1)}(\varepsilon)}{(m+1) !} \prod_{k=0}^{m}\left(x-x_{k}\right) .
$$

Estimates of (23) are considered by the following lemma that have been proved in Yi [18].

Lemma 1 ([18]) If $\varphi(x, t) \in C^{m+1}([a, b])$, then the following estimates for function $e(x)$ defined in (23) hold

$$
\left\{\begin{aligned}
|e(x)| & \leqslant C\left(\frac{e(b-a)}{2 m}\right)^{m}, \\
\left|e_{x}(x)\right| & \leqslant C\left(\frac{e(b-a)}{2(m-1)}\right)^{m-1}, \\
\left|e_{x x}(x)\right| & \leqslant C\left(\frac{e(b-a)}{2(m-2)}\right)^{m-2}
\end{aligned}\right.
$$

For the function $\varphi(x, t)$, we define the error function as follows.

$$
\begin{aligned}
e(x, t)= & \varphi(x, t)-p\left(x_{m}, t_{n}\right) \\
=\frac{\varphi^{(m+1)}\left(\varepsilon_{1}, t\right)}{(m+1) !} \prod_{k=0}^{m}\left(x-x_{k}\right) & \\
& \quad \frac{\varphi^{(n+1)}\left(x, \varepsilon_{2}\right)}{(n+1) !} \prod_{k=0}^{n}\left(t-t_{k}\right) .
\end{aligned}
$$

Based on Lemma 1, we get the following approximation properties.

Theorem 1 For the consistency analysis $e(x, t)$, and the function $\varphi(x, t) \in C^{m+1}[a, b] \times C^{n+1}[0, T]$, we have

$$
\begin{gathered}
|e(x, t)| \leqslant C\left(\frac{e(b-a)}{2 m}\right)^{m}+C\left(\frac{e T}{2 n}\right)^{n}, \\
\left|e_{x}(x, t)\right| \leqslant C\left(\frac{e(b-a)}{2(m-1)}\right)^{m-1}+C\left(\frac{e T}{2 n}\right)^{n}, \\
\left|e_{x x}(x, t)\right| \leqslant C\left(\frac{e(b-a)}{2(m-2)}\right)^{m-2}+C\left(\frac{e T}{2 n}\right)^{n} .
\end{gathered}
$$

In order to give the consistency analysis of the numerical scheme of the barycentric interpolation collocation method, firstly we define the operator

$$
D:=\frac{\partial}{\partial t}-v \frac{\partial^{2}}{\partial x^{2}} .
$$

and let $\varphi\left(x_{m}, t_{n}\right)$ be the numerical solution of $\varphi(x, t)$.

From the above results, we can get the consistency analysis of the barycentric interpolation collocation method as follows.

Theorem 2 If $\varphi \in C^{m+1}[a, b] \times C^{n+1}[0, T]$, we have

$$
\begin{aligned}
& \left|\varphi(x, t)-\varphi\left(x_{m}, t_{n}\right)\right| \leqslant \\
& \quad C\left[\left(\frac{e(b-a)}{2(m-2)}\right)^{m-2}+\left(\frac{e T}{2(n-1)}\right)^{n-1}\right] .
\end{aligned}
$$

Proof: From Eq. (3), we have

$$
\begin{aligned}
& D \varphi(x, t)-D \varphi\left(x_{m}, t_{n}\right) \\
& =\varphi_{t}(x, t)-v \varphi_{x x}(x, t)-\left[\varphi_{t}\left(x_{m}, t_{n}\right)-v \varphi_{x x}\left(x_{m}, t_{n}\right)\right] \\
& =\varphi_{t}(x, t)-\varphi_{t}\left(x_{m}, t_{n}\right)-v\left[\varphi_{x x}(x, t)-\varphi_{x x}\left(x_{m}, t_{n}\right)\right] \\
& =R_{1}-R_{2},
\end{aligned}
$$

where

$$
\begin{aligned}
& R_{1}=\varphi_{t}(x, t)-\varphi_{t}\left(x_{m}, t_{n}\right), \\
& R_{2}=v\left[\varphi_{x x}(x, t)-\varphi_{x x}\left(x_{m}, t_{n}\right)\right] .
\end{aligned}
$$

For $R_{1}$, we have

$$
\begin{aligned}
R_{1} & =\varphi_{t}(x, t)-\varphi_{t}\left(x_{m}, t_{n}\right) \\
& =\varphi_{t}(x, t)-\varphi_{t}\left(x_{m}, t\right)+\varphi_{t}\left(x_{m}, t\right)-\varphi_{t}\left(x_{m}, t_{n}\right) \\
& =e_{t}\left(x, t_{n}\right)+e_{t}\left(x_{m}, t_{n}\right),
\end{aligned}
$$

where $\varphi_{t}\left(x_{m}, t\right)$ indicates that Lagrange interpolation is used only for the spatial direction. Moreover,

$$
\begin{aligned}
\left|R_{1}\right| & \leqslant\left|e_{t}\left(x, t_{n}\right)+e_{t}\left(x_{m}, t_{n}\right)\right| \\
& \leqslant C_{1}\left(\frac{e(b-a)}{2 m}\right)^{m}+C_{2}\left(\frac{e T}{2(n-1)}\right)^{n-1} .
\end{aligned}
$$

Similarly, for $R_{2}$, we have

$$
\begin{aligned}
R_{2} & =v\left[\varphi_{x x}(x, t)-\varphi_{x x}\left(x_{m}, t_{n}\right)\right] \\
& =v\left[e_{x x}\left(x, t_{n}\right)+e_{x x}\left(x_{m}, t_{n}\right)\right],
\end{aligned}
$$

and

$$
\begin{aligned}
\left|R_{2}\right| & \leqslant\left|v e_{x x}\left(x, t_{n}\right)+v e_{x x}\left(x_{m}, t_{n}\right)\right| \\
& \leqslant C_{1}^{*}\left(\frac{e(b-a)}{2(m-2)}\right)^{m-2}+C_{2}^{*}\left(\frac{e T}{2 n}\right)^{n} .
\end{aligned}
$$


Combining the Eqs. (29) and (31), we have

$$
\begin{aligned}
&\left|R_{1}+R_{2}\right| \leqslant C_{1}\left(\frac{e(b-a)}{2 m}\right)^{m}+C_{2}\left(\frac{e T}{2(n-1)}\right)^{n-1} \\
&+C_{1}^{*}\left(\frac{e(b-a)}{2(m-2)}\right)^{m-2}+C_{2}^{*}\left(\frac{e T}{2 n}\right)^{n} \\
& \leqslant C\left(\left(\frac{e(b-a)}{2(m-2)}\right)^{m-2}+\left(\frac{e T}{2(n-1)}\right)^{n-1}\right),
\end{aligned}
$$

where

$$
C:=2 \max \left(C_{1}, C_{2}, C_{1}^{*}, C_{2}^{*}\right) .
$$

It can be seen from the error estimate that the numerical scheme is exponentially convergent, and the order of the differential operator determines the order of convergence of the algebraic equation.

\section{NUMERICAL EXPERIMENTS}

In this section, two examples are provided to verify the high accuracy and numerical stability of the proposed scheme. We define the symbol of the maximum absolute error as follows.

$$
E_{\infty}=\|U-u\|_{\infty},
$$

where $U$ and $u$ denote the numerical solution and the exact solution of the problem, respectively, and $\|\cdot\|_{\infty}$ is the $L^{\infty}$ norm. In the following table, $M+1$ is the number of nodes in space and $N+1$ is the number of time nodes.

Example 1 Consider the Burgers equation with the condition

$$
u(0, t)=u(1, t)=0, \quad 0<t \leqslant T,
$$

and

$$
u(x, 0)=\frac{2 v \pi \sin \pi x}{\alpha+\cos \pi x}, \quad 0<x<1,
$$

where $\alpha>1$. Let $\alpha=2$ in this example. The exact solution of this problem is

$$
u(x, t)=\frac{2 v \pi \mathrm{e}^{-\pi^{2} v t} \sin \pi x}{\alpha+\mathrm{e}^{-\pi^{2} v t} \cos \pi x} .
$$

The initial value condition obtained by Hopf-Cole transformation is

$$
\varphi(x, 0)=\alpha+\cos \pi x, \quad 0 \leqslant x \leqslant 1 .
$$

The boundary conditions are

$$
\left\{\begin{aligned}
\theta(t) \varphi 0, t)+2 v \varphi_{x}(0, t) & =0, & & 0<t \leqslant T \\
\omega(t) \varphi(1, t)+2 v \varphi_{x}(1, t) & =0, & & 0<t \leqslant T .
\end{aligned}\right.
$$

Table 1 The error of barycentric interpolation collocation method and two difference schemes.

\begin{tabular}{ccccc}
\hline & BICM & & CN & CD \\
$(M, N)$ & $E_{\infty}$ & $(M, N)$ & $E_{\infty}$ & $E_{\infty}$ \\
\hline$(5,5)$ & $8.9060 \mathrm{E}-05$ & $(10,10)$ & $5.77030 \mathrm{E}-04$ & $1.9456 \mathrm{E}-05$ \\
$(8,8)$ & $6.4717 \mathrm{E}-07$ & $(20,20)$ & $1.47316 \mathrm{E}-04$ & $2.4010 \mathrm{E}-06$ \\
$(10,10)$ & $4.8313 \mathrm{E}-09$ & $(40,40)$ & $3.70799 \mathrm{E}-05$ & $3.2562 \mathrm{E}-07$ \\
$(13,13)$ & $1.7696 \mathrm{E}-13$ & $(80,80)$ & $9.29913 \mathrm{E}-06$ & $5.7168 \mathrm{E}-08$ \\
$(15,15)$ & $1.5479 \mathrm{E}-14$ & $(160,160)$ & $2.32793 \mathrm{E}-06$ & $1.5802 \mathrm{E}-08$ \\
\hline
\end{tabular}

Table 2 Error norms under different $v$ in Example 1.

\begin{tabular}{lccc}
\hline$v$ & $L^{1}$ & $L^{2}$ & $L^{\infty}$ \\
\hline 2 & $2.1808 \mathrm{E}-03$ & $2.8150 \mathrm{E}-04$ & $1.0755 \mathrm{E}-04$ \\
1 & $1.0734 \mathrm{E}-06$ & $1.4084 \mathrm{E}-07$ & $5.4031 \mathrm{E}-08$ \\
0.1 & $1.2956 \mathrm{E}-11$ & $3.7933 \mathrm{E}-12$ & $3.0918 \mathrm{E}-12$ \\
0.01 & $7.4383 \mathrm{E}-13$ & $9.7579 \mathrm{E}-14$ & $3.7290 \mathrm{E}-14$ \\
0.001 & $1.4404 \mathrm{E}-13$ & $2.2732 \mathrm{E}-14$ & $1.8889 \mathrm{E}-14$ \\
0.0001 & $6.2295 \mathrm{E}-15$ & $5.6827 \mathrm{E}-16$ & $1.3024 \mathrm{E}-16$ \\
\hline
\end{tabular}

Table 1 shows the error comparison of the barycentric interpolation collocation (BICM), Crank-Nicolson (CN), and compact finite difference methods (CD) when $v=0.01$ and $T=1$. It can be seen that the barycentric interpolation collocation method reaches the high precision, as it only uses $10 \times 10$ mesh nodes can achieve the accuracy of compact difference method with $80 \times 80$ mesh nodes. It reflects the advantages of the barycentric interpolation collocation method.

Choose $M=N=15$ for different viscosity coefficients. Table 2 indicates that as $v$ decreases, the error gradually decreases. This shows that when $v$ is small, the barycentric interpolation collocation method can maintain the stability of the numerical solution.

Fig. 1 shows the numerical solution and the exact solution by the barycentric interpolation collocation method. It can be seen from Fig. 1 that the numerical solution and the exact solution are very consistent. Fig. 2(a) shows the absolute error at $M=20, N=10$ and $v=0.01, T=1$. Fig. 2(b) shows the convergence order of the three numerical schemes when $v=0.01$. The figure shows that the barycentric interpolation collocation method converges exponentially while the CrankNicolson converges in the second order and the compact difference method converges in the fourth order. It indicates that the barycentric interpolation collocation method can reach the higher accuracy. Fig. 3(a) shows the numerical solution when final time $T=1,4,8,16$, and Fig. 3(b) shows the numerical solution for $\operatorname{Re}=10,20,50,100(\operatorname{Re}=1 / v)$. 
(a)

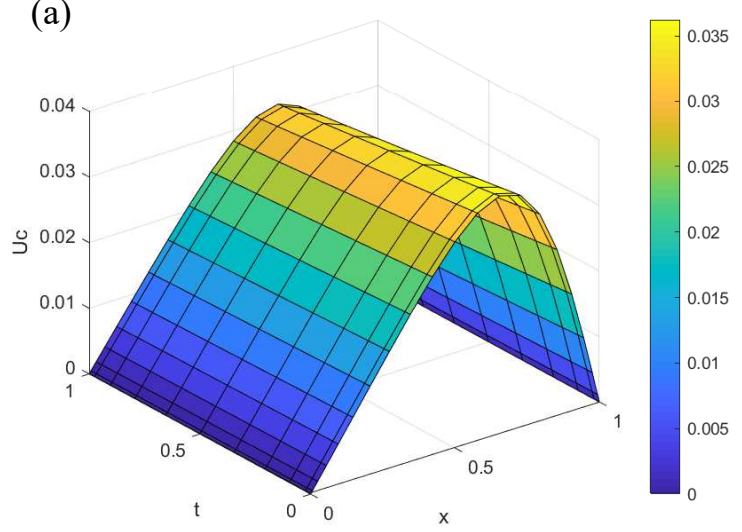

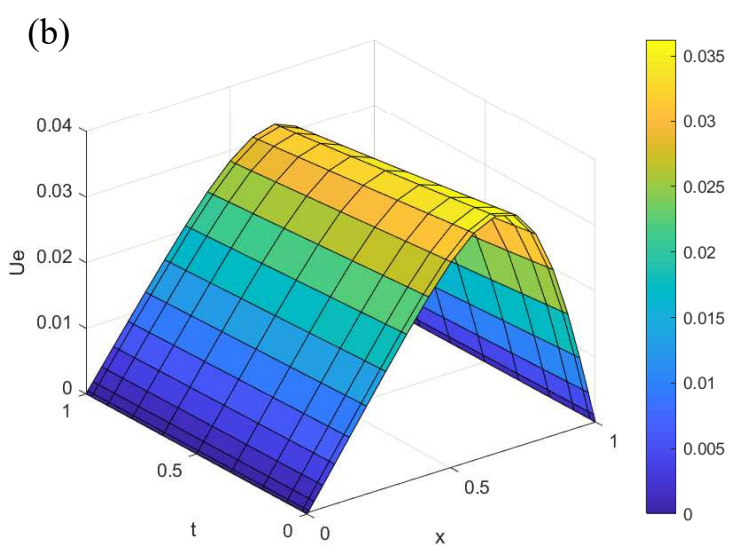

Fig. 1 Numerical solution (a) and exact solution (b) of Example 1 at $M=20, N=10$ and $v=0.01$.
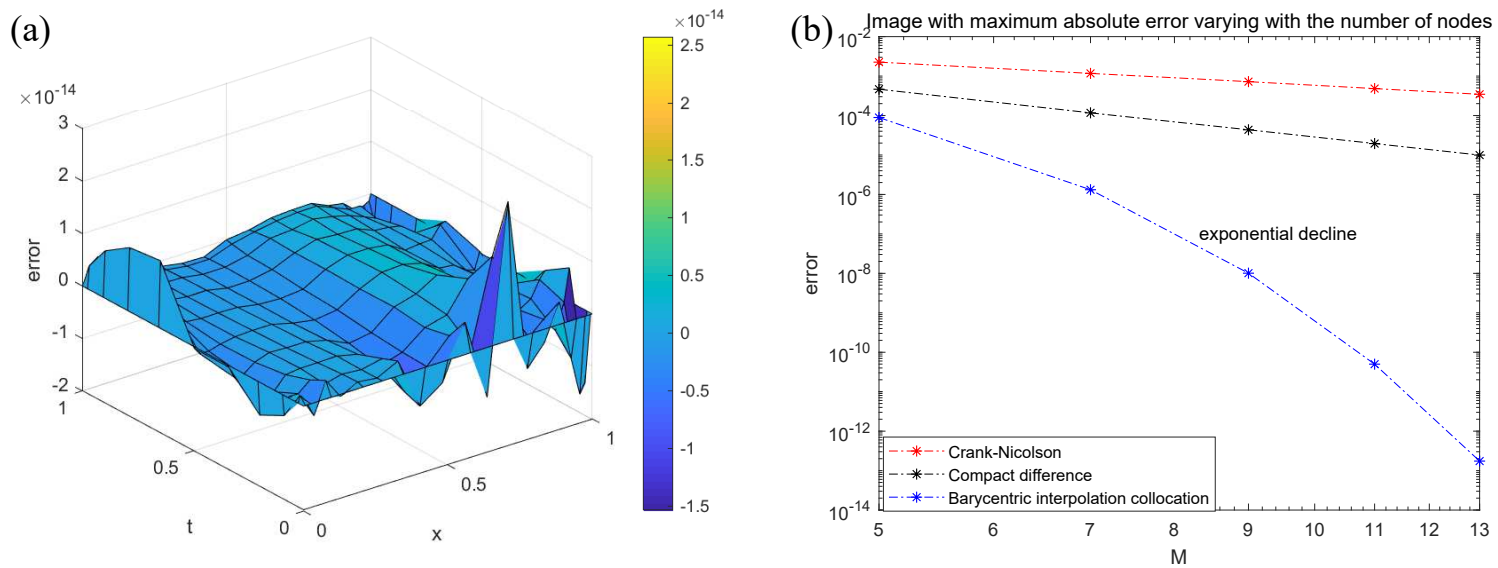

Fig. 2 (a) The absolute error at $M=20, N=10$, and $v=0.01$ and (b) convergence rate of Example 1.

(a)

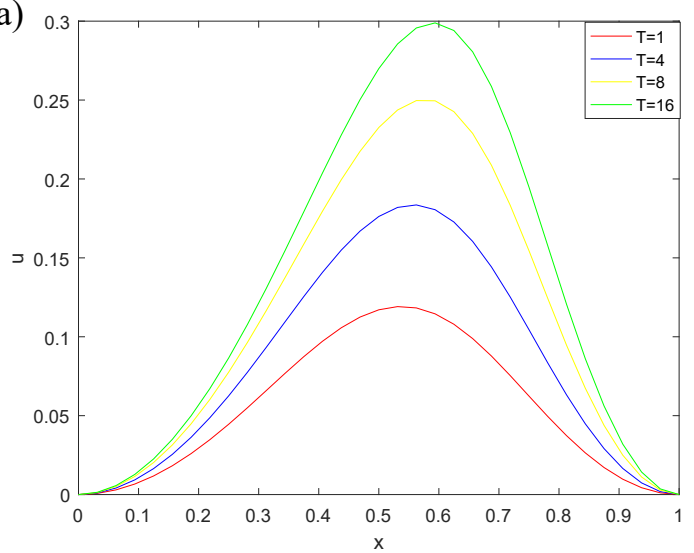

(b)

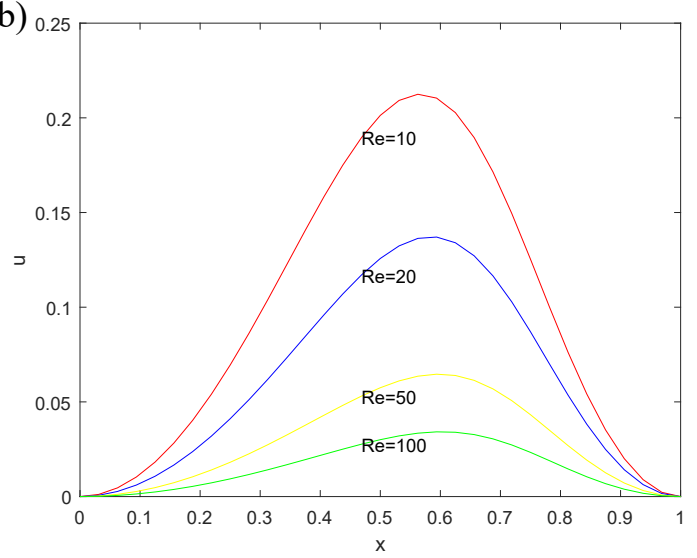

Fig. 3 Numerical solution of the model under different $T$ (a) and Re (b) of Example 1 at $M=N=32$. 

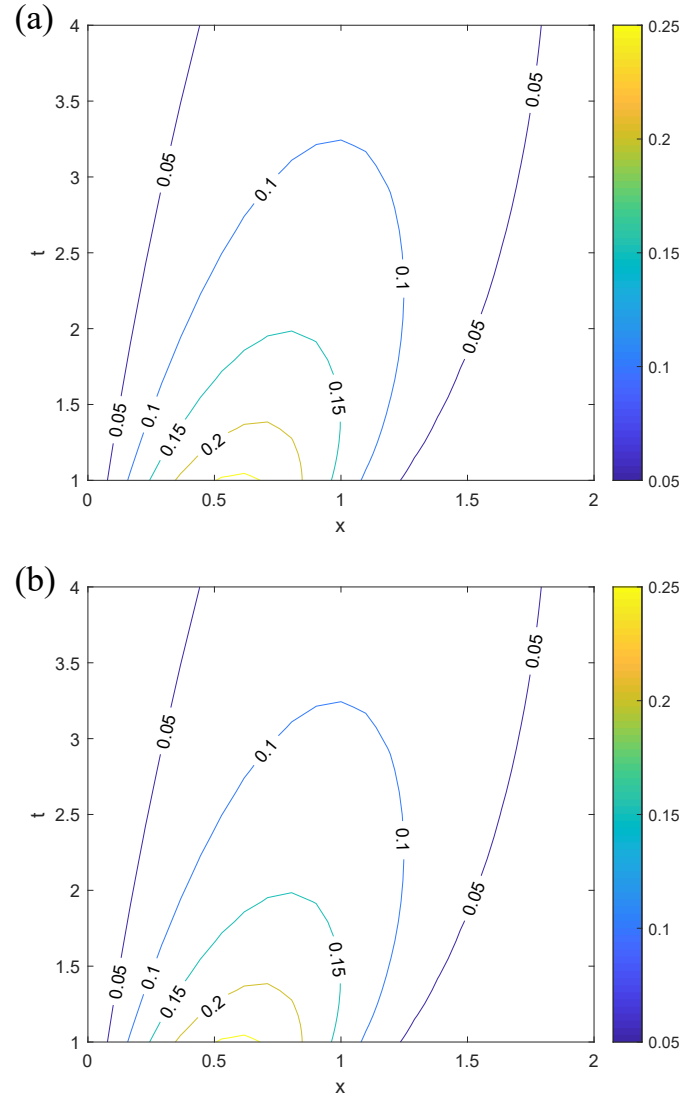

Fig. 4 Numerical solution (a) and exact solution (b) of Example 2 at $M=N=32$ and $v=0.1$.

Table 3 The error of barycentric interpolation collocation method and two difference schemes.

\begin{tabular}{lclcc}
\hline & BICM & & $\mathrm{CN}$ & $\mathrm{CD}$ \\
$(M, N)$ & $E_{\infty}$ & $(M, N)$ & $E_{\infty}$ & $E_{\infty}$ \\
\hline$(15,15)$ & $1.4926 \mathrm{E}-06$ & $(10,10)$ & $3.20999 \mathrm{E}-03$ & $2.6151 \mathrm{E}-03$ \\
$(18,18)$ & $1.2589 \mathrm{E}-08$ & $(20,20)$ & $9.04298 \mathrm{E}-04$ & $3.1646 \mathrm{E}-04$ \\
$(20,20)$ & $1.7086 \mathrm{E}-09$ & $(40,40)$ & $2.40032 \mathrm{E}-04$ & $8.3901 \mathrm{E}-05$ \\
$(23,23)$ & $1.4039 \mathrm{E}-11$ & $(80,80)$ & $6.18768 \mathrm{E}-05$ & $3.4015 \mathrm{E}-05$ \\
$(25,25)$ & $1.0839 \mathrm{E}-12$ & $(160,160)$ & $1.57064 \mathrm{E}-05$ & $1.6054 \mathrm{E}-05$ \\
\hline
\end{tabular}

Example 2 Consider the following Burgers equation for $0<x<2,1 \leqslant t \leqslant T$,

$$
u(x, t)=\frac{x / t}{1+\sqrt{t / t_{0}} \exp \left(x^{2} / 4 \varepsilon t\right)} .
$$

The initial value condition obtained by Hopf-Cole transformation is

$$
\begin{aligned}
\varphi(x, 1)=\exp \left(-\frac{x^{2}}{4 \varepsilon}+\ln (1+\sqrt{1 / t}\right. & \left.\left.\exp \left(x^{2} / 4 \varepsilon\right)\right)\right), \\
0 & <x<2 . \quad(40)
\end{aligned}
$$

(a)

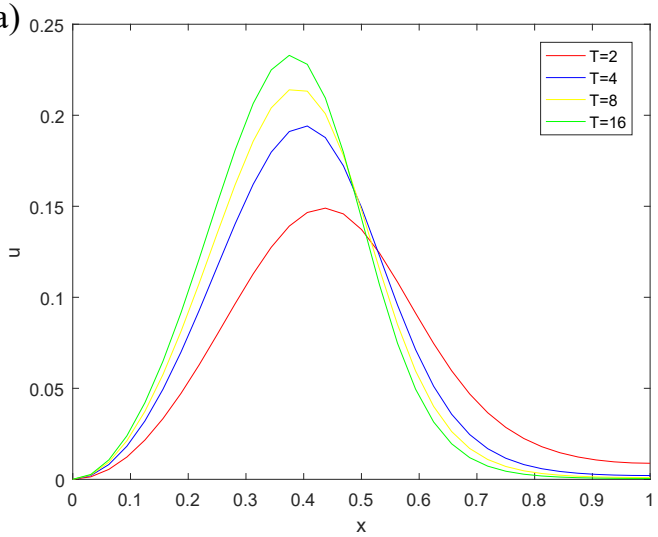

(b)

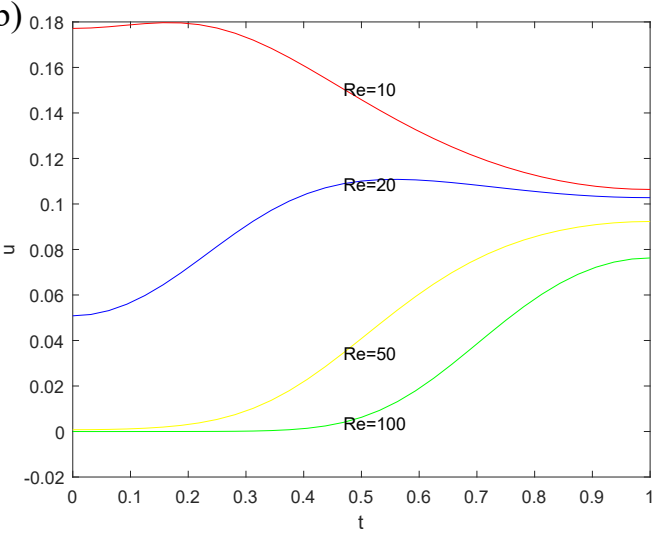

Fig. 5 Numerical solution of different $T$ (a) and Re (b) of Example 2 at $M=N=32$.

The boundary conditions are

$$
\left\{\begin{aligned}
\theta(t) \varphi(0, t)+2 v \varphi_{x}(0, t) & =0, & & 1<t<T \\
\omega(t) \varphi(2, t)+2 v \varphi_{x}(2, t) & =0, & & 1<t<T .
\end{aligned}\right.
$$

Table 3 shows the error of the barycentric Lagrange interpolation collocation, Crank-Nicolson, and compact difference methods when $v=0.01$ and $T=2$. From Table 3, it can be seen that, compared with the Crank-Nicolson and compact finite difference methods, the barycentric Lagrange interpolation collocation method can achieve the higher accuracy.

Fig. 4 shows the contour of the numerical solution and the exact solution. Fig. 5(a) shows the numerical solution when final time $T=2,4,8,16$, and Fig. 5(b) shows the numerical solution for $R e=$ $10,20,50,100(\operatorname{Re}=1 / v)$. Fig. 6 shows the absolute error at $M=N=25, v=0.1$, and $T=2$. 


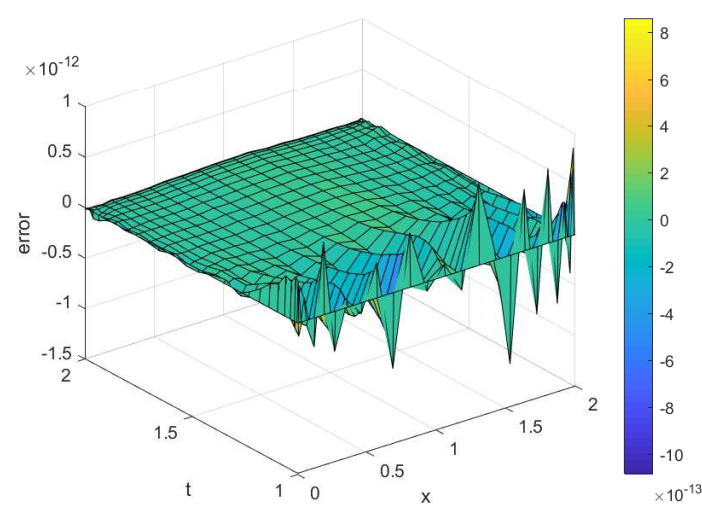

Fig. 6 Absolute error of Example 2 at $M=N=25$ and $v=0.1$.

\section{CONCLUSION}

In this paper, with the help of Hopf-Cole transformation and barycentric interpolation collocation method, an efficient numerical scheme for Burgers equation is developed. Besides, the consistency analysis for the barycentric interpolation collocation method is derived. Numerical examples show that our proposed scheme can achieve the higher accuracy with small nodes and error results are consistent with the analysis. In future work, we plan to extend the barycentric interpolation collocation method for the high-dimensional Burgers equations and combine it with other methods to discuss the high-order numerical scheme of the Burgers equation.

Acknowledgements: This work is in part supported by the Natural Science Foundation of China (No. 11701197), the Fundamental Research Funds for the Central Universities (No. ZQN-702), the Key Laboratory of Intelligent Computing and Information Processing of Ministry of Education (No. 2020ICIP03) and supported by the Innovation Training Project of Fujian Province (S202010385043) and Student Science and Technology Innovation Key Projects of Huaqiao university (XS-19).

\section{REFERENCES}

1. Bateman H (1915) Some recent researches on the motion of fluids. Mon Weather Rev 43, 163-170.

2. Hopf E (1950) The partial differential equation $u_{t}+$ $u u_{x}=u_{x x}$. Commun Pure Appl Math 3, 201-230.

3. Kadalbajoo MK, Awasthi A (2006) A numerical method based on Crank-Nicolson scheme for Burgers equation. Appl Math Comput 182, 1430-1442.
4. Jiwari R (2015) A hybrid numerical scheme for the numerical solution of the Burgers equation. Comput Phys Commun 188, 59-67.

5. Zhang Q, Wang X, Sun Z (2020) The point-wise estimates of a conservative difference scheme for Burgers equation. Numer Methods Partial Differ Equ 36, 1611-1628.

6. Sari M (2019) Higher order splitting approaches in analysis of the Burgers equation. Kuwait J Sci 46, $1-14$.

7. Wang HF, Xu D, Zhou J, Guo J (2021) Weak Galerkin finite element method for a class of time fractional generalized Burgers equation. Numer Methods Partial Differ Equ 37, 732-749.

8. Berrut JP, Trefethen LN (2004) Barycentric Lagrange interpolation. SIAM Rev 46, 501-517.

9. Higham NJ (2004) The numerical stability of barycentric Lagrange interpolation. IMA $J$ Numer Anal 24, 547-556.

10. Floater MS, Hormann K (2007) Barycentric rational interpolation with no poles and high rates of approximation. Numer Math 107, 315-331.

11. Tian D, He JH (2018) The barycentric rational interpolation collocation method for boundary value problems. Therm Sci 22, 1773-1779.

12. Zhang Y, Zhang W, Zhao C, Wang Y (2020) Numerical solution of a coupled reaction-diffusion model using barycentric interpolation collocation method. Therm Sci 24, 2561-2567.

13. Li M, Huang C (2019) The linear barycentric rational quadrature method for auto-convolution Volterra integral equations. J Sci Comput 78, 549-564.

14. Liu H, Huang J, Pan Y, Zhang J (2018) Barycentric interpolation collocation methods for solving linear and nonlinear high-dimensional Fredholm integral equations. $J$ Comput Appl Math 327, 141-154.

15. Azin H, Mohammadi F, Baleanu D (2020) A generalized barycentric rational interpolation method for generalized Abel integral equations. Int $J$ Appl Comput Math 6, ID 140.

16. Luo WH, Huang TZ, Gu XM, Liu Y (2017) Barycentric rational collocation methods for a class of nonlinear parabolic partial differential equations. Appl Math Lett 68, 13-19.

17. Wang YL, Tian D, Li ZY (2017) Numerical method for singularly perturbed delay parabolic partial differential equations. Therm Sci 21, 1595-1599.

18. Yi SC, Yao LQ (2019) A steady barycentric Lagrange interpolation method for the 2D higher order timefractional telegraph equation with nonlocal boundary condition with error analysis. Numer Methods Partial Differ Equ 35, 1694-1716.

19. Li J, Cheng Y (2021) Linear barycentric rational collocation method for solving heat conduction equation. Numer Methods Partial Differ Equ 37, 533-545. 